\title{
Properties of bcr-abl-transformed mouse 12B1 cells secreting interleukin-2 and granulocyte-macrophage colony-stimulating factor: I. Derivation, genetic stability, oncogenicity and immunogenicity
}

\author{
MARTINA PETRÁČKOVÁ, RUTH TACHEZY and VLADIMÍR VONKA \\ Department of Experimental Virology, Institute of Hematology and Blood Transfusion, \\ U Nemocnice 1, Prague 128 20, Czech Republic
}

Received November 17, 2011; Accepted December 29, 2011

DOI: 10.3892/ijo.2012.1365

\begin{abstract}
The highly oncogenic bcr-abl-transformed mouse (Balb/c) 12B1 cells were transfected with plasmids carrying genes for either mouse interleukin-2 (IL-2) or the mouse granulocyte-macrophage colony-stimulating factor (GM-CSF) and the gene for blasticidine resistance. From the transduced cells several clones widely differing in the production of either cytokine were isolated. For further experiments, clones with the highest secretion of the cytokines were selected. When administered subcutaneously to mice, the IL-2-secreting cell line was approximately hundred times less pathogenic than the parental cells. A portion of animals developed small, spontaneously regressing tumours and most of them became resistant to challenge with the parental cells. Cell populations from either solid tumours or from organs infiltrated by the tumour cells predominantly consisted of cells which did not produce IL-2 and had lost resistance to blasticidine. This indicated that the IL-2 secreting cells were genetically unstable in the course of their propagation in vivo. On the other hand, the GM-CSF-secreting cells were more pathogenic than the parental cells, induced extensive organ damage and remained genetically stable in the course of their growth in vivo. The pathogenicity of different GM-CSF secreting clones directly depended on the magnitude of production of this cytokine. When used in the form of inactivated vaccines, the GM-CSF-secreting cells were more immunogenic than the IL-2-secreting cells. In comparative experiments, similar results were obtained with GM-CSF- and IL-2-secreting cells derived from B210 cells, another bcr-abl transformed cell line.
\end{abstract}

Correspondence to: Dr Vladimír Vonka, Department of Experimental Virology, Institute of Hematology and Blood Transfusion, U Nemocnice 1, Prague 128 20, Czech Republic

E-mail:vonka@uhkt.cz

Key words: chronic myeloid leukaemia, bcr-abl, gene-modified cells, pathogenicity, immunogenicity

\section{Introduction}

Numerous studies have shown that cellular vaccines engineered to secrete various immunostimulatory cytokines are capable of surpassing the poor immunogenicity of most tumour-associated antigens and can generate a potent, specific and long-lasting anti-cancer immunity. Thus, the use of geneengineered whole-cell vaccines is gradually developing into a promising approach in immunotherapy. Their augmented immunogenicity is apparently due to local coupling of cytokine production and antigen presentation. The lack of serious untoward reactions, as well as the fact that the host is exposed to the whole spectrum of antigens, this securing the involvement of the immunologically most important ones, provides further support for this concept.

Of the immunostimulatory cytokines, attention has especially been paid to interleukin-2 (IL-2) and the granulocyte-macrophage colony-stimulating factor (GM-CSF). IL-2 secreting cellular vaccines have repeatedly been used with partial success in both preclinical and clinical studies (1-14). GM-CSF secreted by gene-engineered tumour cells has been found to be the most potent stimulatory factor among the ten cytokines tested (15) and it has since become the most frequently used cytokine in the construction of cell-based cancer vaccines. GM-CSF secreting vaccines have been used in numerous preclinical and clinical studies, for reviews see (16-18).

Although most clinical studies have dealt with solid tumours, there seems to be a growing interest in utilizing gene-modified cells for the treatment of chronic myeloid leukaemia (CML) and other haematological malignancies. Both autologous and allogenic cell vaccines are under consideration $(17,19)$. Favourable results have been obtained in postremission acute myeloid leukaemia patients with a vaccine composed of a mixture of autologous cells and K562 cells, i.e. a cell line derived from a CML patient, which were engineered to secrete GM-CSF (20). Encouraging results have been shown in lethally irradiated GM-CSF-secreting autologous cells for the treatment of acute myeloid leukaemia and myelodysplasia patients who had received allogenic stem cell transplantations (21). Quite recently, it was further shown in a preclinical 
model that the administration of a GM-CSF-secreting myeloid leukaemia cellular vaccine prior to autologous bone marrow transplantation significantly prolonged the survival of mice (22). Recently, also a therapeutic vaccine based on GM-CSFsecreting K562 cells was successfully used for the treatment of CML patients (23)

In the past few years we tried to obtain some more information on immune reactions to bcr-abl-transformed cells in the murine system. The key element of these studies was the development of a variety of vaccines aimed at inducing protection against challenge with these cells $(24,25)$ and at testing their immunotherapeutic potential (13). In the previous paper of ours (26), we showed that bcr-abl-transformed mouse (Balb/c) B210 cells, which had been gene-modified by transfection of mouse genes for IL-2, IL-12 or GM-CSF immunostimulatory factors, had lost their capability of inducing leukaemia in syngeneic animals. It was of interest to determine how a similar genetic modification would influence the pathogenicity and immunogenicity of another mouse (Balb/c) bcr-abl-transformed cell line, namely 12B1 cells, which are more oncogenic than the B210 cells after intravenous administration and, in addition, form solid lymphoma-like tumours after subcutaneous (s.c.) administration.

In this study we explored the properties of 12B1 cells expressing murine IL-2 and GM-CSF proteins. We also tested their immunogenic potency and compared it with that induced by similarly modified B210 cells.

\section{Materials and methods}

Cell lines and media. 12B1 is a murine leukaemia cell line derived by the transformation of Balb/c bone marrow cells with a retrovirus-derived vector carrying the human bcr-abl (b3a2) fusion gene (27). The 12B1 cells were kindly provided by Dr E. Katsanis (University of Arizona, Tuscon, AZ). Their in vitro and in vivo properties have been described in more detail elsewhere $(28,29)$. They have the phenotype of early pre-B cells, are CD19 positive and induce leukaemialike disease in mice after intravenous administration. In our hands, $1 \mathrm{TID}_{50}$ corresponds to approximately $10^{2}$ cells. After s.c. inoculation $12 \mathrm{~B} 1$ cells induce solid tumours, with $1 \mathrm{TID}_{50}$ corresponding to approximately $10^{2.5}$ cell. The cells were cultivated in RPMI-1640 medium (Sigma-Aldrich Corp., St. Louis, MO) supplemented with $10 \%$ heat-inactivated fetal calf serum (FCS) (PAA Laboratories, Linz, Austria), 4 mM glutamine, $1 \mathrm{mM}$ sodium pyruvate, $50 \mu \mathrm{M} 2$-mercaptoethanol, penicillin $(100 \mathrm{U} / \mathrm{ml})$ and streptomycin $(100 \mu \mathrm{g} / \mathrm{ml})$, at $37^{\circ} \mathrm{C}$ in $5 \% \mathrm{CO}_{2}$ atmosphere. For the selection of cells successfully transduced with plasmids (see below), the medium was supplemented with blasticidine (25 $\mu \mathrm{g} / \mathrm{ml})$ (InvivoGen, San Diego, CA). To obtain cTK-less cells, we cultivated 12B1 cells in RPMI-1640 medium with a gradually increasing concentration of 5-bromo-2-deoxyuridine (BrdU) (Sigma-Aldrich). The 12B1 TK-less cells grew well at a concentration of $100 \mu \mathrm{g} \mathrm{BrdU} / \mathrm{ml}$. The derivation and properties of IL-2- and GM-CSF-secreting B210 cells, designated B210/2/IL-2/cl-21 (producing $30 \mathrm{ng}$ of the cytokine per $10^{6}$ cells/24 h) and B210/2/GM-CSF/cl-1 (producing $40 \mathrm{ng}$ of the cytokine per $10^{6}$ cells $/ 24 \mathrm{~h}$ ) have been described elsewhere (26). These cells were derived from clone no. 2 isolated from the population of B210 TK-less cells, which was the most pathogenic of the TK-less clones tested. B210/2 will be referred to as parental cells. All of the B210 derived cells were passaged in RPMI supplemented with $10 \%$ FCS and enriched with hypoxanthin-aminopterin-thymidine (HAT) (HAT Supplement, Gibco, Invitrogen, Carlsbad, CA). The cells were kept in liquid nitrogen. In all experiments the third passage of rethawed cells was used. 293T cells (kindly provided by Dr J. Kleinschmidt, DKFZ, Heidelberg, Germany) were used as a negative control in Western blotting. Their propagation was the same as in previous experiments (8).

Plasmids. Plasmids pBSC/IL-2 (30) and pBSC/GM-CSF were used. Both plasmids were constructed and kindly provided by Dr M. Šmahel (Institute of Hematology and Blood Transfusion, Prague). The latter plasmid was constructed using pBKGM (31). From this plasmid, cDNA of mouse GM-CSF was cut out and ligated into the pBSC plasmid (Dr M. Šmahel, unpublished data). Plasmids pBSC/IL-2 and pBSC/GM-CSF were linearized with $\mathrm{SacII}$ restriction enzyme (New England Biolabs, Beverley, MA). The resulting ends were treated with T4-DNA polymerase (Promega, Madison, WI) and NotI restriction enzyme (New England Biolabs) creating one blunt and one NotI end. To supplement the plasmids with a gene for a selection marker, we isolated a fragment from pBLAST42mAngio (InvivoGen, San Diego, CA) containing the SV40 promoter, the gene for blasticidine resistance (BsrS2) and the poly A end. First we linearized the plasmid with $B s r$ GI restriction enzyme (New England Biolabs). The ends were treated with Klenow fragment (New England Biolabs) and then with NotI restriction enzyme (New England Biolabs) to produce one blunt and one NotI overlapping end. This fragment was then ligated into linearized pBSC/IL-2 and pBSC/GM-CSF plasmids. The new constructs were denoted $\mathrm{pBSC} / \mathrm{IL}-2-\mathrm{Bsr}$ and $\mathrm{pBSC} / \mathrm{GM}-\mathrm{Bsr}$, respectively. The plasmid pTR-UF2 (32) carrying gene for green fluorescent protein (GFP) was used for monitoring the transfection efficiency. Plasmids were propagated in Escherichia coli DH5- $\alpha$ strain (Gibco) and purified using the Maxi Prep DNA Isolation Qiagen kit (Qiagen, Hilden, Germany). For the experiment with 12B1 TK-less cells, we used bicistronic plasmids pTRGM-CSF-IRES-TK and pTR-IL2-IRES-TK (8).

Electroporation. Before electroporation, plasmids $\mathrm{pBSC} /$ GM-Bsr and pBSC/IL-2-Bsr were linearized with KpnI enzyme (New England Biolabs). Plasmids pTR-UF2, pTR-GM-CSF-IRES-TK and pTR-IL-2-IRES-TK were used in non-linearized form. Electroporation was performed using the Gene Pulser Electroporation system (Bio-Rad, Hercules, CA) as described previously (26). Electroporation was carried out at room temperature exposing the cells to $280 \mathrm{~V}$, $1050 \mu \mathrm{F}$. The cells were then transferred into tissue culture dishes containing $4 \mathrm{ml}$ of regular cultivation media. After $48 \mathrm{~h}$ cultivation the cells were spun down and resuspended in the respective RPMI-1640 medium. Cells transfected with pBSC/GM-Bsr and pBSC/IL-2-Bsr were cultivated in media supplemented with $25 \mu \mathrm{g} / \mathrm{ml}$ of blasticidine, those transfected with the HSV TK gene-carrying plasmids in media with HAT and those transfected with pTR-UF2 in regular media. The transfection efficiency was determined by flow cytometric analysis of GFP expression in 12B1 cells transfected with plasmid pTR-UF2 $24 \mathrm{~h}$ after electroporation. 
Cell cloning. To isolate cell clones from transduced cells, fresh cultivation medium was mixed with conditioned medium, at a 4:1 ratio. The conditioned medium was obtained from the culture of the particular transfected cells at its logarithmic growth phase. Before mixing it with the fresh medium, the conditioned medium was filtered through a $0.22 \mathrm{~mm}$-SyringeFilter (TPP, Trasadingen, Switzerland). To isolate cell clones, transfected cells were diluted to the final concentration of 0.3 cell $/ 0.2 \mathrm{ml}$ and distributed in $0.2 \mathrm{ml}$ aliquots into a 96-well plate (TPP). Four h after seeding, all wells were carefully checked for the presence of cells and those containing only one cell were marked. After about 14 day incubation the cell suspensions from these wells were transferred into bigger plates. Again, the cell lines derived were kept frozen in liquid nitrogen until being used and the third passage of rethawed cells was used.

Animal experiments. Six to 8 weeks old female Balb/c mice were obtained from Charles Rivers, Germany. All experiments were carried out in accordance with the Guidelines for Animal Experimentation valid in the Czech Republic. For oncogenicity tests and for challenge, cells were washed 3 times with PBS and the appropriate counts of cells in $0.2 \mathrm{ml}$ of PBS were injected s.c. When applied as vaccines in immunization/challenge experiments, the cells were inactivated using $\gamma$-radiation (100 Gy) (IBL 437C irradiator ${ }^{137} \mathrm{Cs}$-irradiation source, CIS Bio International, Gif-Sur-Yvette Cedex, France), washed 3 times in PBS, and injected in $0.2 \mathrm{ml}$ of PBS intraperitoneally. Gene-modified B210 cells, which had lost their pathogenicity for mice, were used as live vaccines. In both cases two doses of $3 \times 10^{6}$ cells were administered at a two-week interval, and 2 weeks later the animals were challenged with $5 \times 10^{3}$ parental 12B1 cells. Mice were monitored 3 times a week for up to 90 days. When tumours reached the size of $400 \mathrm{~mm}^{2}$, the mice were humanely sacrificed. Mice which did not develop a tumour and manifested symptoms of leukaemia-like disease were sacrificed when impaired vitality, limited mobility, bristled hair and/or hind-leg paresis were observed.

Generation of cell cultures from tumours or organs infiltrated by tumour cells. Tumours or selected organs were excised from the mice under sterile conditions. They were mechanically disrupted in small volumes of complete RPMI medium to produce cell suspensions, filtered through a cell strainer and centrifuged. The cell pellets were resuspended in regular media and cultivated. Third passage cultures were split into media with or without blasticidine. Their growth activity and cytokine production (see below) were monitored.

$R T$-PCR. To detect bcr-abl-expressing cells RT-PCR was performed as described previously (12), except that the concentration of RNA was determined with Nanodrop (Nanodrop Technologies, Wilmington, DE). Spleens, livers and bone marrows were tested.

Measurement of cytokine production. Concentrations of cytokines in cell culture media were measured by ELISA using the BD OptEIA ${ }^{\mathrm{TM}}$ set mouse GM-CSF or mouse IL-2 kits (BD Biosciences, San Diego, CA), following the manufacturer's instructions. Counts of $1 \times 10^{6}$ cells were seeded in
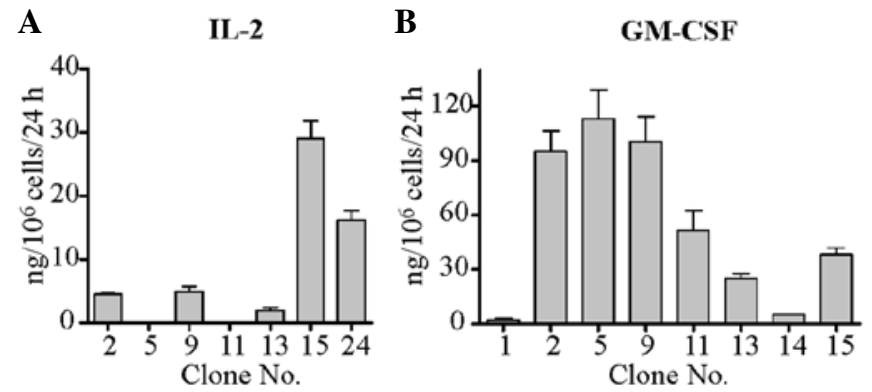

Figure 1. Cytokine production by cell clones derived from $12 \mathrm{~B} 1$ cells transduced by plasmids carrying genes for (A) mouse IL-2 and (B) mouse GM-CSF. The data are from 3 independent experiments.

$3 \mathrm{ml}$ medium in $6-\mathrm{cm}$ culture dishes. After $24 \mathrm{~h}$ cells were counted and spun down and the supernates were tested for the content of the cytokines. Cell viability was determined using the trypan blue exclusion test. The production level was calculated according to the formula: $\mathrm{C} / \mathrm{N}$ (where $\mathrm{C}$ is the total amount of cytokine in culture medium and $\mathrm{N}$ is the final viable cell count) and was expressed in $\mathrm{ng} / 10^{6}$ cells $/ 24 \mathrm{~h}$.

Western blotting. Cell lysates were prepared and Western blotting with anti-c-ABL monoclonal antibody (Ab-3, Oncogene Research Products, Boston, MA) was performed as described previously (26). Then the membrane was washed 2 times in PBS with $0.1 \%$ Tween and reprobed with anti- $\beta$-tubulin monoclonal antibody (Sigma-Aldrich, Steinheim, Germany).

Flow cytometry. Flow cytometry for monitoring GFP-expressing cells was performed on the Beckman Coulter EPICS XL. For analysis of the results WinMDI 2.8 software was used.

Statistical analysis. For analysis of survival the log-rank test and for the analysis of tumour growth two-way ANOVA test were used. Calculations were done using Prism software version 5.0 (Graph-Pad Software, San Diego, CA).

\section{Results}

Isolation of genetically modified $12 \mathrm{Bl}$ cells. Originally, we tried to isolate gene-modified 12B1 cells similarly to that performed in B210 cells (26). Thus, our plan was to isolate TK-less cells by repeated passages in the presence of increasing concentrations of BrdU, transfect them with plasmids carrying genes for the respective cytokines and HSV TK and select the transduced cells in HAT media. These early experiments confronted us with two unexpected outcomes. First, when compared with the isolation of B210 TK-less cells, which involved many passages requiring more than 6 months, the isolation of 12B1 TK-less cells, i.e. cells growing well in the presence of $100 \mu \mathrm{g}$ BrdU, was easily achieved in a few weeks. Second, when these cells were transfected with plasmids carrying the HSV TK gene, cells growing in HAT media were selected readily; however, cells growing well in the same media also appeared in cultures of mock-transfected cells. Apparently, revertants to the $\mathrm{TK}^{+}$phenotype were present in the populations of the putative TK-less 12B1 cells. Both observations, i.e. easy isolation of TK-less cells and their rapid reversion, demonstrated a 
A

12B1/GM-CSF/cl-1

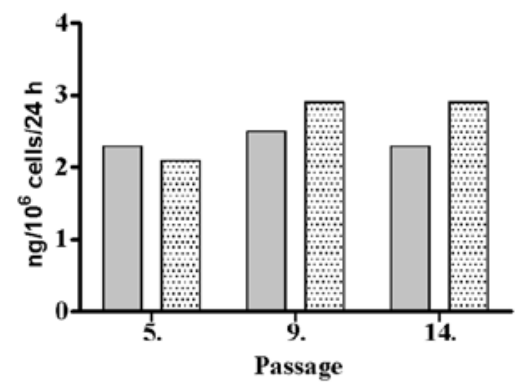

B

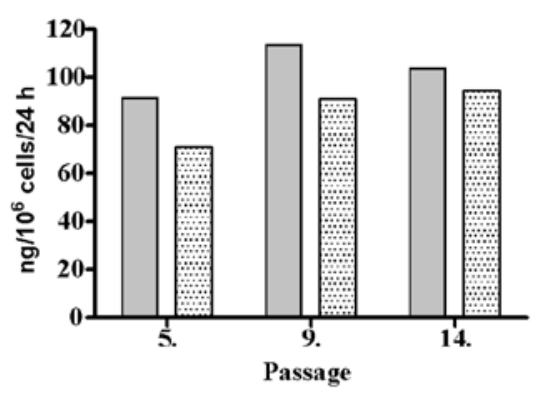

C

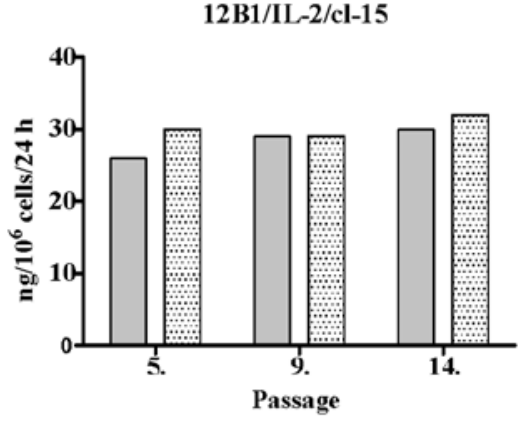

w/o blasticidine

with blasticidine

Figure 2. Stability of expression of the respective cytokines in the course of passages in vitro either in the presence or absence of blasticidine. Clones (A) 12B1/ GM-CSF/cl-1, (B) 12B1/GM-CSF/cl-5 and (C) 12B1/IL-2/cl-15 were tested.

genetic instability of the 12B1 cells and suggested that another approach had to be used for the isolation of the gene-modified cells.

For this purpose we constructed plasmids carrying either the IL-2 or the GM-CSF gene and the blasticidine resistance gene, as described in Materials and methods. Cells were transfected by electroporation; the efficiency was determined by flow cytometry measuring GFP-positive 12B1 cells after transfection with the pTR-UF2 plasmid. In repeated tests, 12 to $18 \%$ of live cells were GFP+. A total of 24 clones and a total of 12 clones were isolated from the populations of the IL-2-transfected and GM-CSF-transfected cells, respectively, growing in blasticidine containing selective media. Seven clones of pBSC/IL-2-Bsr-transfected cells and 8 clones of pBSC/GM-Bsr-transfected cells were tested for the production of the respective cytokines. The production was expressed in $\mathrm{ng} / 10^{6}$ cells $/ 24 \mathrm{~h}$. As indicated in Fig. 1, all of the GM-CSFtransfected clones produced the cytokine but only 5 out of 7 IL-2-transfected clones were positive. Furthermore, individual clones markedly differed in the magnitude of production.

For subsequent experiments we selected clone no. 15 for IL-2-secreting cells, designated 12B1/IL-2/cl-15, and clones no. 5 and no. 1 for the GM-CSF-secreting cells, designated 12B1/GM-CSF/cl-5 and 12B1/GM-CSF/cl-1. Before their inoculation into animals, we determined the stability of the cytokine production in vitro by passaging these cells in the presence or absence of blasticidine. The results are summarized in Fig. 2. It is evident that the extent of production remained unaltered in both the selective and non-selective media, this proving the in vitro genetic stability of the transduced cell lines and also the lack of influence of the product of the blasticidine resistance gene on the growth of the transduced cells in the non-selective medium.

Western blotting. The results of Western blotting are shown in Fig. 3. They indicate that there were no marked differences in the expression of bcr-abl protein among the cell lines tested.

Pathogenicity of transduced cells. Groups of mice were inoculated s.c. with $10^{3}-10^{6}$ of the gene-modified cells. Mice inoculated with $10^{3}$ and $10^{4}$ of the parental 12B1 cells served as controls. The results are summarized in Fig. 4. All mice inoculated with $10^{6} 12 \mathrm{~B} 1 / \mathrm{IL}-2 / \mathrm{cl}-15$ cells died before day 45 .
A

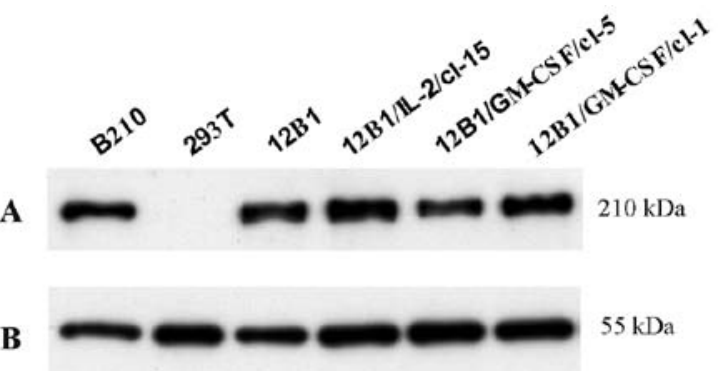

Figure 3. Production of bcr-abl protein by $12 \mathrm{~B} 1$ cells and their gene-modified cell lines, as determined by Western blot analysis. Protein lysates were separated in $7 \%$ gel, transferred onto PVDF membrane and treated with anti-c-ABL monoclonal antibody to detect $\mathrm{p} 210^{\mathrm{bcr}-\mathrm{abl}}$ protein (A). The same membrane was reprobed with anti- $\beta$-tubulin monoclonal antibody (B). B210 cells served as a positive and $293 \mathrm{~T}$ cells as a negative control.

It may be of interest that all animals in this group developed small subcutaneous tumours, which regressed by day 25 and later on all of them died of leukaemia. Their death was markedly delayed when compared with mice, which had been inoculated with parental 12B1 cells in doses 100 and 1000 times lower. Without exception animals inoculated with the parental cells developed rapidly growing subcutaneous tumours. Mice inoculated with $10^{5}$ 12B1/IL-2/cl-15 cells also developed small subcutaneous tumours, which regressed by day 21. One mouse developed a big tumour later on and the other two mice survived. All animals inoculated with the $10^{4}$ and $10^{3} \mathrm{IL}-2$-secreting cells remained healthy throughout the observation period, this indicating that the secretion of IL-2 was associated with attenuation of the cells. The surviving animals were challenged on day 79 with $10^{5} 12 \mathrm{~B} 1$ cells, i.e. with approximately $300 \mathrm{TID}_{50}$, given s.c. Four of 7 mice inoculated survived this indicating that the inoculation of a rather small amount of 12B1/IL-2/cl-15 cells was capable of eliciting protection against challenge with a high dose of the parental cells in the majority of animals. After additional 75 days, the survivors were rechallenged with the same dose of 12B1 cells. Three of 4 mice remained healthy and were free of bcr-abl-positive cells in their bone marrow, liver and spleen, as demonstrated by RT-PCR (results not shown). Similar results were obtained in the repeated experiment, in which 9 out of 10 surviving animals remained healthy after challenge given 
A

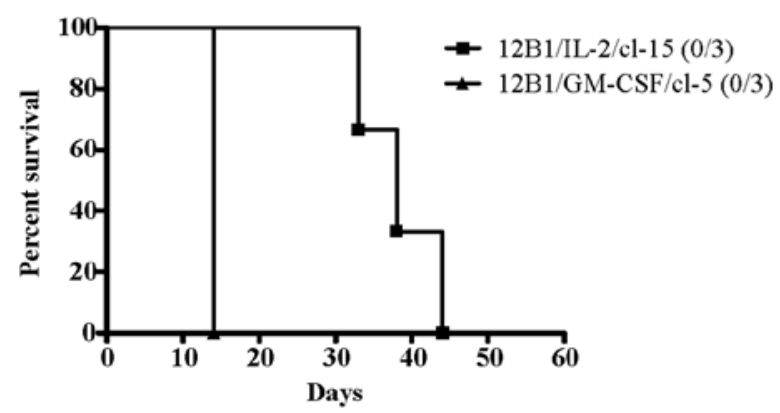

C

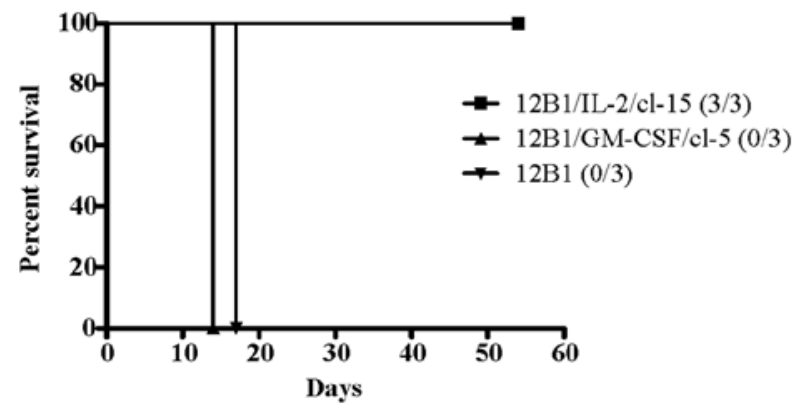

B

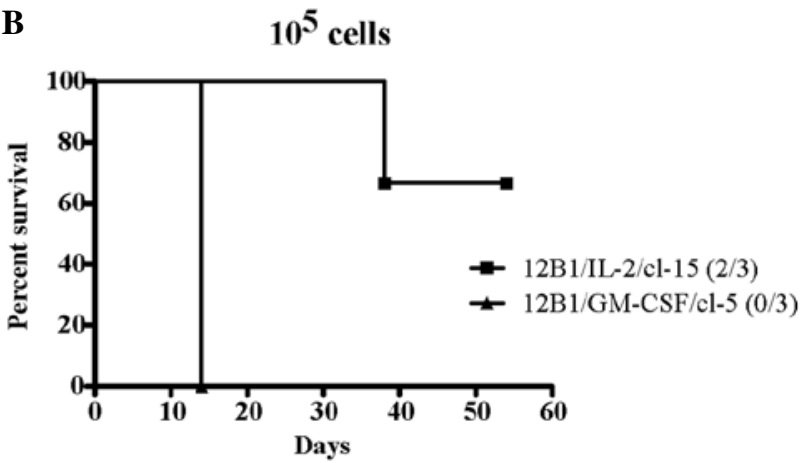

D
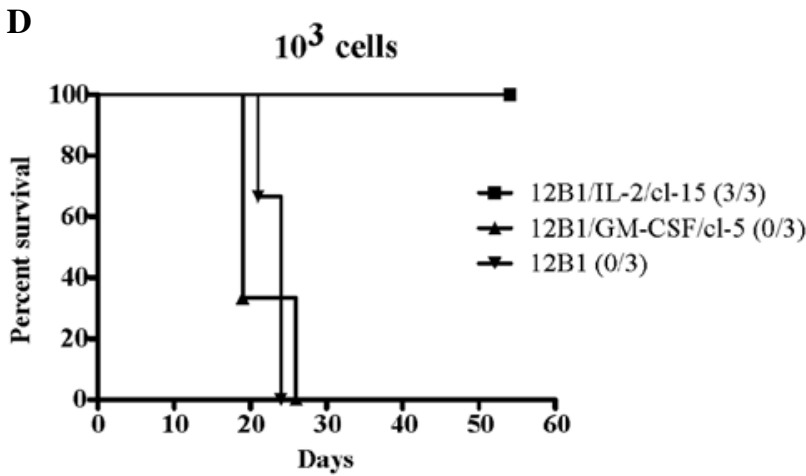

Figure 4. Pathogenicity of 12B1-derived cell lines designated 12B1/IL-2/cl-15 and 12B1/GM-CSF/cl-5 and parental 12B1 cells. Mice were inoculated s.c. with: (A) $10^{6}$, (B) $10^{5}$, (C) $10^{4}$ and (D) $10^{3}$ cells.

on day 75. On the other hand, all animals inoculated with 12B1/GM-CSF/cl-5 cells developed tumours similar to those induced by the control parental 12B1 cells. In addition, these animals had bristled hair and showed a loss of weight, and their autopsy revealed extensive organ damage never seen in mice inoculated with the parental 12B1 cells (33). Similar results were obtained in repeated experiments.

The induction of tumours by the gene-modified cells provided us with a possibility to test the genetic stability of the cells under investigation in vivo. Several tumours that

Table I. Cytokine production of the cell suspensions derived from mice inoculated with 12B1/IL-2/cl-15 and with 12B1/GM-CSF/ cl-5 cells.

\begin{tabular}{|c|c|c|c|c|c|}
\hline Cells inoculated & Mouse no. & Day of autopsy & Source of cells & Passage & Production of cytokine \\
\hline \multirow[t]{5}{*}{ 12B1/IL-2/cl-15 } & 1 & 38 & Tumour & $\begin{array}{c}3 \\
2+3 \mathrm{Bl}^{\mathrm{b}}\end{array}$ & $\begin{array}{c}0.6 \\
40\end{array}$ \\
\hline & 2 & 44 & Spleen & $\begin{array}{c}3 \\
2+4 \mathrm{Bl}\end{array}$ & $\begin{array}{c}0.7 \\
50\end{array}$ \\
\hline & & & Liver & $\begin{array}{c}2 \\
2+4 \mathrm{Bl}\end{array}$ & $\begin{array}{l}0.5 \\
20\end{array}$ \\
\hline & & & Blood & $\begin{array}{c}2 \\
2+4 \mathrm{Bl}\end{array}$ & $\begin{array}{l}2.7 \\
18\end{array}$ \\
\hline & 3 & 49 & Tumour & $\begin{array}{c}4 \\
2+6 \mathrm{Bl}\end{array}$ & $\begin{array}{c}0 \\
25\end{array}$ \\
\hline \multirow[t]{2}{*}{ 12B1/GM-CSF/cl-5 } & 4 & 19 & Tumour & $\begin{array}{c}3 \\
2+4 \mathrm{Bl}\end{array}$ & $\begin{array}{l}127 \\
115\end{array}$ \\
\hline & 5 & 23 & Tumour & $\begin{array}{c}3 \\
2+4 \mathrm{Bl}\end{array}$ & $\begin{array}{l}120 \\
111\end{array}$ \\
\hline
\end{tabular}

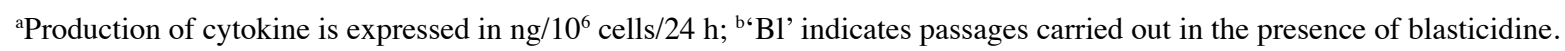


A

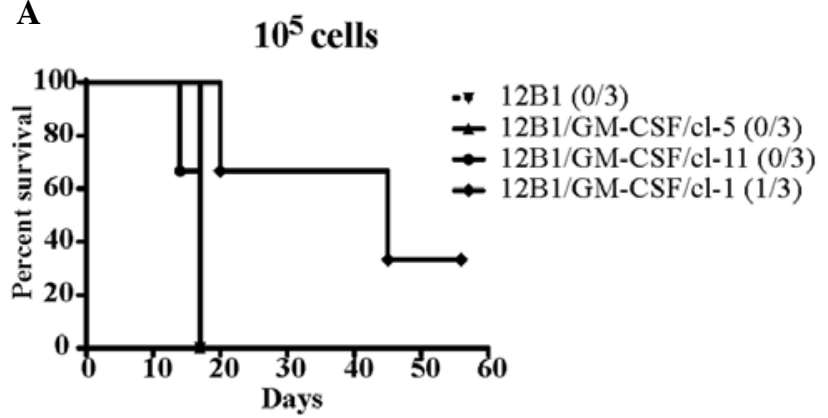

B $10^{3}$ cells

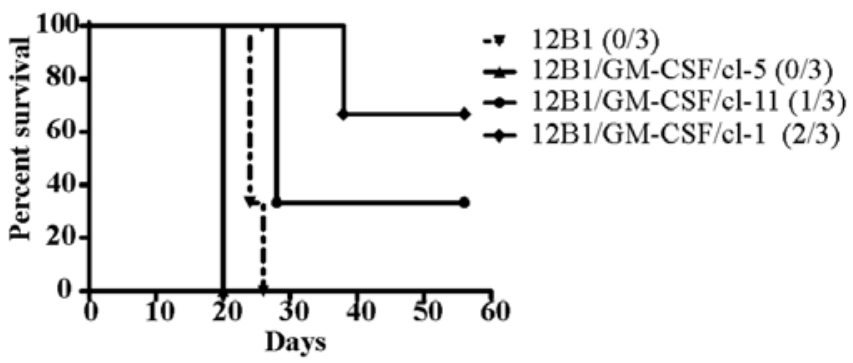

Figure 5. Pathogenicity of 12B1-derived cell lines differing in the production of GM-CSF; 12B1/GM-CSF/cl-5: $110 \mathrm{ng} / 10^{6} \mathrm{cells} / 24 \mathrm{~h} ; 12 \mathrm{~B} 1 / \mathrm{GM}-\mathrm{CSF} /$ cl-11: $50 \mathrm{ng} / 10^{6}$ cels $/ 24 \mathrm{~h} ; 12 \mathrm{~B} 1 / \mathrm{GM}-\mathrm{CSF} / \mathrm{cl}-1: 3 \mathrm{ng} / 10^{6}$ cells $/ 24 \mathrm{~h}$. Mice were inoculated s.c. with (A) $10^{5}$ and (B) $10^{3}$ cells.

had developed in mice after the administration of the genemodified cells were surgically removed, and cell suspensions were mechanically prepared. In one animal inoculated with 12B1/IL-2/cl-15 cells, which did not develop a solid tumour but leukaemia, cell cultures were prepared from its liver, spleen and blood. Cells were cultivated in parallel in media either containing or not-containing blasticidine. The data shown in Table I demonstrate marked differences between the cells derived from the $12 \mathrm{~B} 1 / \mathrm{IL}-2 / \mathrm{cl}-15$ and the $12 \mathrm{~B} 1 /$ GM-CSF/cl-5 inoculated mice. Initially, 12B1/IL-2/cl-15 derived cells grew poorly in the presence of blasticidine and produced low amounts of IL-2 both in the presence and absence of the antibiotic. However, after a few passages in its presence, blasticidine-resistant cells were readily selected and they proved to be efficient producers of the cytokine. These findings demonstrate the instability of 12B1/IL-2/cl-15 cells in vivo. Clearly, the cell populations of the tumour cells and the populations of cells infiltrating various organs were not homogeneous, being composed of a great majority of cells in which the two transgenes were not expressed, either owing to their loss or silencing, and a minority of transduced cells. The results obtained with cells derived from 12B1/GM-CSF/ cl-5 induced tumours were dramatically different. From the very beginning, the cells grew very well in the presence of blasticidine and already the first passage cells secreted high amounts of GM-CSF, comparable with its production by the cells inoculated.

To examine the relationship between GM-CSF production and cell pathogenicity, we tested, in parallel, three GM-CSFsecreting cell clones markedly differing in the production of the cytokine. In addition to $12 \mathrm{~B} 1 / \mathrm{GM}-\mathrm{CSF} / \mathrm{cl}-5$, producing $110 \mathrm{ng}$ GM-CSF $/ 10^{6}$ cells $/ 24 \mathrm{~h}$, clones denoted $12 \mathrm{~B} 1 / \mathrm{GM}-\mathrm{CSF} /$ cl-11 producing $50 \mathrm{ng} / 10^{6} \mathrm{cells} / 24 \mathrm{~h}$ and $12 \mathrm{~B} 1 / \mathrm{GM}-\mathrm{CSF} / \mathrm{cl}-1$

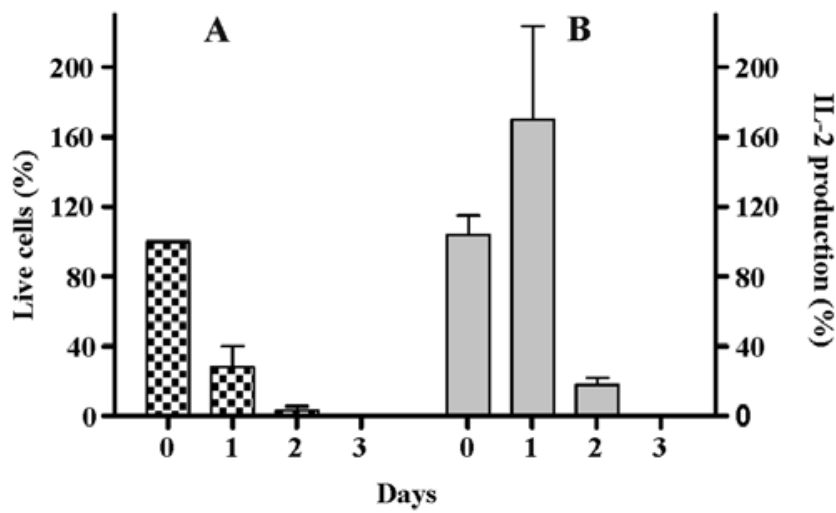

Figure 6. Survival of and cytokine production by irradiated (100 Gy) 12B1/ IL-2/cl-15 cells. The total of $3 \times 10^{6}$ of irradiated cells was placed in cultivation media. At $24 \mathrm{~h}$ intervals the cell suspension was spun down, (A) the viability of the cells and (B) the cytokine contents in the supernates were determined and the cells were resuspended in fresh media. The data originate from 3 independent experiments.

producing only $3 \mathrm{ng} / 10^{6}$ cells $/ 24 \mathrm{~h}$ of the cytokine, were tested. The results are shown in Fig. 5. While at a dose of $10^{5}$ cells all animals except those inoculated with the lowest cytokine producer, i.e. 12B1/GM-CSF/cl-1 cells, died of leukaemia before their tumour reached the critical size justifying euthanasia, a more pronounced difference became apparent after the administration of $10^{3}$ cells. The most efficient producer of the cytokine, 12B1/GM-CSF/cl-5 cells, was the most pathogenic, causing death of the animals more rapidly than the parental 12B1 cells or clones producing lesser amounts of the cytokine. Clearly 12B1/GM-CSF/cl-1 cells, with the lowest production of GM-CSF, were the least pathogenic. These observations suggested a relationship between GM-CSF production and pathogenicity. It may be of interest that mice, which survived both the high and the low cell dose administered, were free of bcr-abl-expressing cells, as determined by RT-PCR, at the end of the observation period (data not shown).

Efficacy of irradiated gene-modified cells in immunization/ challenge experiments. Next, the efficacy of irradiated genemodified 12B1 cells as tumour vaccines was investigated. Since we realized that the vaccine efficacy would depend on the ability of the irradiated cells to secrete the cytokine, before using them as vaccines we tested the viability of the irradiated cells by the trypan blue exclusion test and their capability of secreting the respective cytokine. As shown in Fig. 6, the irradiated 12B1/IL-2/cl-15 cells were dead within $48 \mathrm{~h}$. After an initial increase, the cytokine production rapidly dropped in parallel with their dying and virtually stopped after $48 \mathrm{~h}$. The same phenomenon was observed in all cell lines tested irrespective of whether they secreted IL-2 or GM-CSF. Irradiated 12B1/GM-CSF/cl-5 cells, 12B1/IL-2/cl-15 cells and the parental 12B1 cells ( $3 \times 10^{6} /$ mouse) were injected intraperitoneally twice at a 2-week interval and challenged as described in Materials and methods. As shown in Fig. 7A, all animals vaccinated with irradiated 12B1/GM-CSF/cl-5 cells remained tumour-free throughout the observation period, whereas 2 out of 6 mice vaccinated with irradiated 12B1/IL-2/cl-15 developed tumours before day 24 after challenge. Vaccination with irradiated 

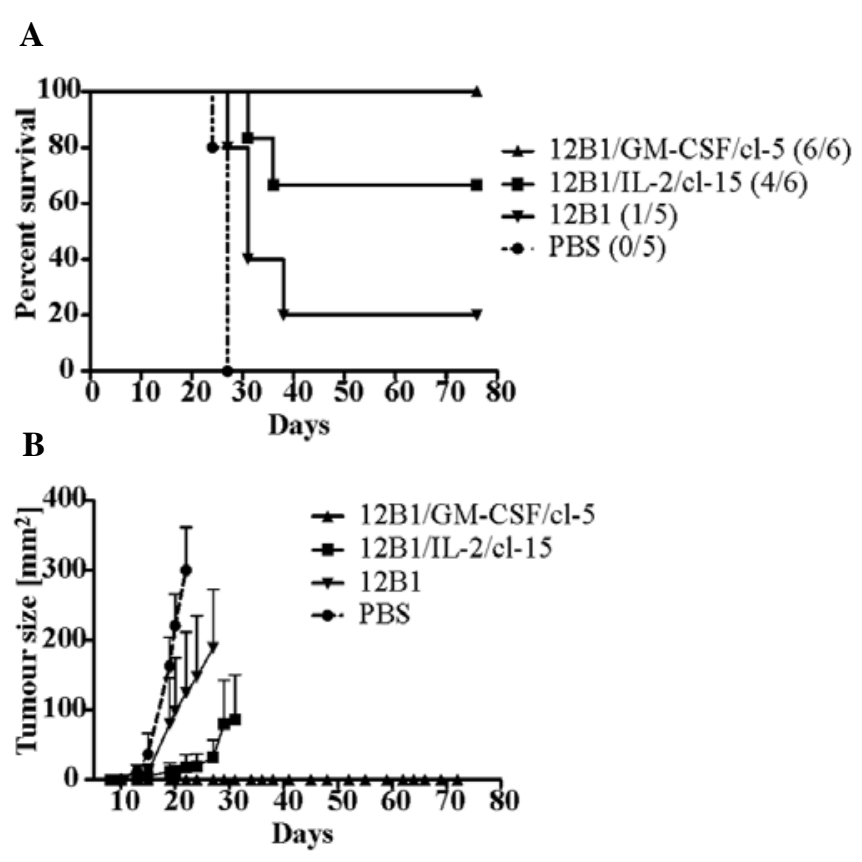

Figure 7. Immunogenicity of irradiated 12B1/GM-CSF/cl-5, 12B1/IL-2/cl-15 and parental 12B1 cells. Mice were immunized i.p. with two doses of $3 \times 10^{6}$ cells in 2-week interval. Two weeks after the second dose the animals were challenged s.c. with $5 \times 10^{3}$ parental $12 \mathrm{~B} 1$ cells and monitored for (A) survival and (B) tumour growth. Graph (B) represents mean tumour size \pm SEM. Statistics: Survival: 12B1/GM-CSF/cl-5 vs. 12B1 or PBS p<0.01; 12B1/IL-2/ cl-15 vs. PBS $p<0.01 ; 12 B 1$ vs. PBS $p<0.02$, the other differences are NS. Tumour growth: The growth trends of all the groups are mutually different with high significance $(\mathrm{p}<0.001)$. The experiment was repeated with similar results.

parental cells was even less effective: 4 out of 5 mice developed tumours before day 40. As indicated in Fig. 7B, the tumours in immunized mice grew more slowly than in the non-immunized animals. Surviving animals were rechallenged on day 78 with $5 \times 10^{3}$ of parental $12 \mathrm{~B} 1$ cells. All 6 mice vaccinated with 12B1/GM-CSF/cl-5 cells remained healthy for the subsequent 60 days. Bone marrows, livers and spleens from these animals were checked for the presence of bcr-abl-expressing cells by RT-PCR, with negative results (data not shown). Similar effects of vaccination were observed in a repeated test. In this experiment 12B1/GM-CSF/cl-1-based vaccine was also used. This vaccine was less potent in inducing protective immunity than the vaccine prepared from the high-producer cells but more potent than those prepared from the parental cells or 12B1/ IL-2/cl-15 cells (data not shown).

Since also B210 cells secreting IL-2 and GM-CSF were available, it was of interest to compare the immunogenicity of these cells with their 12B1-derived counterparts. As these cells had completely lost their pathogenicity for mice (26), they were administered in the form of live vaccines, using the same immunization schedule as in the case of gene-engineered 12B1 cells. As challenge, parental 12B1 cells were used. The results are shown in Fig. 8. In general, the immunization effect of B210-based vaccines was less potent than in the case of vaccines based on the homologous, gene-engineered 12B1 cells. However, it was clear again that the GM-CSF-secreting cells were a more potent immunogen that the IL-2-secreting cells. The latter cells failed to induce any protection. Similar results were obtained in a repeated test.
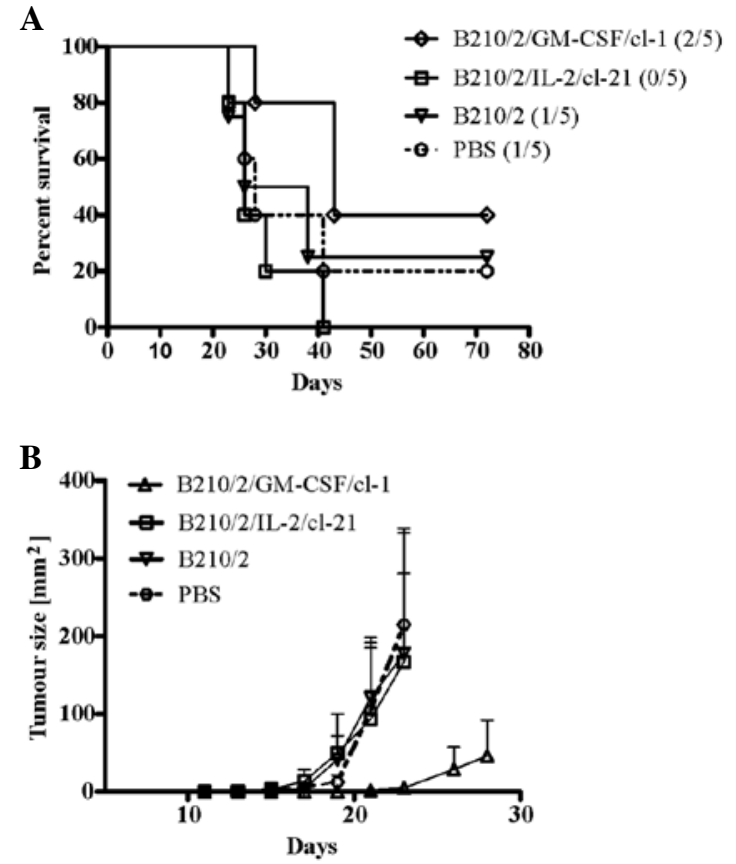

Figure 8. Immunogenicity of live B210/2/GM-CSF/cl-1, B210/2/IL-2/cl-21 and parental B210/2 cells. Mice were immunized in 2-week interval with $3 \times 10^{6}$ cells i.p.. Two weeks after the second immunization animals were challenged s.c. with $5 \times 10^{3}$ parental $12 \mathrm{~B} 1$ cells and monitored for (A) survival and (B) tumour growth. Graph (B) represents mean tumour size \pm SEM. Statistics: Survival: B210/2/GM-CSF/cl-1 vs. B210/2/IL-2/cl-21 p <0.05; the other differences were NS. Tumour growth: B210/2/GM-CSF/cl-1 vs. B210/2 or B210/2/IL-2/cl-21 p $<0.001$, B210/2/GM-CSF/cl-1 vs. PBS p $<0.01$, the other differences are NS. The experiment was repeated with similar results.

\section{Discussion}

One of the main present trends in the immunotherapy of cancer is based on the assumption that gene-engineered tumour cells secreting immunostimulatory cytokines might be effective in the therapy of cancer. In the recent past, employing the bcr-abl-transformed B210 TK-less cells and HSV TK as the selection gene, we derived several gene-modified cell lines producing IL-2, IL-12 or GM-CSF. Invariably, these cells had lost their pathogenicity for mice (26). Possibly even more important were some additional observations which indicated that (i) the administration of these cells resulted in a postponement of tumour formation in mice which had been inoculated with the highly aggressive sister mouse cell line, 12B1, and (ii) the administration of these cells prevented tumour development in a substantial proportion of 12B1-inoculated animals, if combined with cyclophosphamide plus imatinib mesylate and/or interferon $\alpha$ given in doses which per se were incapable of suppressing tumour development (13). In this respect, the IL-2-secreting cells were the most effective. Although both B210 and 12B1 produce comparable amounts of the p210 $10^{\mathrm{bcr}-a b l}$ protein, they differ in a number of other characteristics $(28,29$; Krmenčiková et al, unpublished). Therefore, it was of interest to examine the properties of similarly gene-engineered 12B1 cells. In the present study we report on the properties of 12B1 cells transduced to secrete IL-2 and GM-CSF. Clearly, these cell lines differed considerably from similarly transduced B210 cells. First, another system had to be employed for their derivation. Since the 12B1 TK-less cells proved to be 
genetically unstable, and thus not suitable for the isolation of the transduced cells, plasmids with the HSV TK gene were replaced with plasmids carrying the blasticidine resistance gene for the selection of transduced cells. Second, the transduced cells had not lost their pathogenicity for mice. Still, the 12B1-IL-2-secreting cells were clearly less oncogenic than the parental cells. In a significant proportion of animals the small subcutaneous tumours which had developed, regressed spontaneously and these animals became resistant to challenge with the parental 12B1 cells. Third, the analysis of the tumour cell populations demonstrated a genetic instability of the 12B1-IL-2 cells in vivo, this contrasting with the previously detected genetic stability of the transduced cells in the course of multiple tissue culture passages. In an in vitro analysis of the tumour cell populations, the in vivo instability manifested itself both by a decreased production of the cytokine and increased sensitivity to blasticidine. However, cultivation of these cells in the presence of blasticidine resulted in a rapid selection of the transduced IL-2 producing cells from the tumour cell population. These observations suggested that the cells in which the transgenes had either been lost or silenced had a selective advantage for in vivo growth over those preserving the transduced phenotype. Fourth, the behaviour of the GM-CSF-expressing sublines was very different. These results suggested that, at variance with IL-2-secreting cells, the GM-CSF-secreting cells had a selective advantage in vivo. Their capability of forming subcutaneous tumours was not diminished, and, in addition, the disease induced by these cells was clearly more severe than that induced by the parental cells. Data were obtained suggesting that this extra-pathogenicity depended on the amount of the GM-CSF produced. Subsequent experiments indicated that the growth of the 12B1/GM-CSF/ cl-5 cells in vivo was associated with extensive damage to several organs. These pathological findings are described and discussed separately (33).

Finally, in the present series of experiments, we tested the ability of these cells, used in the form of irradiated vaccines, to induce immunity against challenge with the parental cells While in some other systems the secretion of the cytokines monitored was not compromised by lethal irradiation $(34,35)$ and was reported to continue for up to 5 (31) or even 12 days (36), we observed a quick shut-off of their production in our system. In spite of the apparently short-time secretion in vivo, vaccines based on our gene-engineered cells were clearly more immunogenic than the parental cells. The GM-CSF secreting vaccine proved to be a more potent immunogen than the IL-2 secreting vaccine, which was in agreement with the evidence obtained in other systems in the past (15). Again, there was a relationship between the amount of GM-CSF produced and the immunization effect: the vaccine based on the low-producer 12B1/GM-CSF/cl-1 cells was somewhat less effective than that based on the high producer 12B1/ GM-CSF/cl-5 cells, this underscoring the key role of the cytokine in eliciting the anti-tumour immunity. These data clearly indicated that the high-producer cells, absolutely unsuitable for use as live vaccines, are preferable for the development of inactivated vaccines. In this respect it was of interest to test the efficacy of the similarly engineered B210 cells which, because of complete loss of pathogenicity, could be employed as live attenuated vaccines. In the present study we used them for immunization against the heterologous 12B1 cells. Although one could conjecture that the live vaccines would be more immunogenic than the inactivated ones, the opposite was true, this most likely expressing the difference in the antigenic make-up of B210 and 12B1 cells, but also the lower production of the cytokines, though it was in the case of GM-CSF still above the critical level (37). The protection induced by the B210-GM-CSF-secreting cells was incomplete and none was detected when IL-2-secreting cells were used for immunization. This finding was in contrast with the results obtained when using the same cell lines as therapeutic vaccines. In those experiments, in which the same lines of transduced cells were employed, IL-2-secreting cells acted as a more potent immunogen than the GM-CSF secreting tumour cells (13). Those data may indicate that the quite common trend to gene-engineered therapeutic cancer vaccines to secrete GM-CSF does not need to have general validity. They suggest that the preferences for optimal cytokine treatment may differ owing to a variety factors related to the magnitude of the cytokine secretion, specificities of the various tumour systems but also whether live or non-viable cell vaccines are employed.

\section{Acknowledgements}

The authors thank Kateřina Kernová and Tereza Nováková for excellent technical assistance, Michal Šmahel for kindly providing plasmids and Zdeněk Roth for help with statistical analysis. This work was supported by the grant no. NS-10634-3/2009 of the Internal Granting Agency of the Ministry of Health, Czech Republic and by the Research Project MZOUHKT2005.

\section{References}

1. Orchard PJ, Katsanis E, Boyer M, May C, McIvor RS and Blazar BR: Interleukin-2 secretion by transduced and unselected BDL-2 lymphoma results in increased survival in mice with previously established disseminated disease. Cancer Biother Radiopharm 11: 155-164, 1996.

2. Tung C, Federoff HJ, Brownlee M, Karpoff H, Weigel T, Brennan MF and Fong Y: Rapid production of interleukin-2-secreting tumor cells by herpes simplex virus-mediated gene transfer: implications for autologous vaccine production. Hum Gene Ther 7: 2217-2224, 1996.

3. Rodolfo M, Zilocchi C, Melani C, Cappetti B, Arioli I, Parmiani G and Colombo MP: Immunotherapy of experimental metastases by vaccination with interleukin gene-transduced adenocarcinoma cells sharing tumor-associated antigens. Comparison between IL-12 and IL-2 gene-transduced tumor cell vaccines. J Immunol 157: 5536-5542, 1996.

4. Van EA, Aarnoudse C, van der Minne CE, van der Spek CW, Brouwenstijn N, Osanto S and Schrier PI: Transfection of IL-2 augments CTL response to human melanoma cells in vitro: immunological characterization of a melanoma vaccine. $J$ Immunother 20: 343-353, 1997.

5. Heller L, Pottinger C, Jaroszeski MJ, Gilbert R and Heller R: In vivo electroporation of plasmids encoding GM-CSF or interleukin-2 into existing B16 melanomas combined with electrochemotherapy induces long-term antitumour immunity. Melanoma Res 10: 577-583, 2000.

6. Mazzocchi A, Melani C, Rivoltini L, et al: Simultaneous transduction of B7-1 and IL-2 genes into human melanoma cells to be used as vaccine: enhancement of stimulatory activity for autologous and allogeneic lymphocytes. Cancer Immunol Immunother 50: 199-211, 2001.

7. Ge NL, Ye SL, Zheng N, Sun RX, Liu YK and Tang ZY: Prevention of hepatocellular carcinoma in mice by IL-2 and B7-1 genes co-transfected liver cancer cell vaccines. World J Gastroenterol 9: 2182-2185, 2003. 
8. Jinoch P, Zak R, Janouskova O, et al: Immunization with live HPV-16-transformed mouse cells expressing the herpes simplex thymidine kinase and either GM-CSF or IL-2. Int J Oncol 23 : 775-783, 2003.

9. Indrova M, Bubenik J, Mikyskova R, et al: Chemoimmunotherapy in mice carrying HPV16-associated, MHC class I+ and class I- tumours: Effects of CBM-4A potentiated with IL-2, IL-12, GM-CSF and genetically modified tumour vaccines. Int J Oncol 22: 691-695, 2003

10. Sobotkova E, Duskova M, Smahel M, Holan V, Janouskova O and Vonka V: Chemotherapy and immunotherapy of tumours induced by gene-modified HPV16-transformed cells. Oncol Rep 12: 877-883, 2004.

11. Rittich S, Duskova M, Mackova J, Pokorna D, Jinoch P and Smahel M: Combined immunization with DNA and transduced tumor cells expressing mouse GM-CSF or IL-2. Oncol Rep 13: 311-317, 2005.

12. Sobotkova E, Duskova M, Eckschlager T and Vonka V: Efficacy of reovirus therapy combined with cyclophosphamide and gene-modified cell vaccines on tumors induced in mice by HPV16-transformed cells. Int J Oncol 33: 421-426, 2008.

13. Sobotkova E, Duskova M, Tachezy R, Petrackova M and Vonka V: Combined chemo- and immunotherapy of tumors induced in mice by bcr-abl-transformed cells. Oncol Rep 21: 793-799, 2009.

14. Rossowska J,Pajtasz-PiaseckaE,Rysnik O,Wojas J,Krawczenko A Szyda A and Dus D: Generation of antitumor response by IL-2-transduced JAWS II dendritic cells. Immunobiology 216 1074-1084, 2011

15. Dranoff G, Jaffee E, Lazenby A, et al: Vaccination with irradiated tumor cells engineered to secrete murine granulocytemacrophage colony-stimulating factor stimulates potent, specific and long-lasting anti-tumor immunity. Proc Natl Acad Sci USA 90: 3539-3543, 1993.

16. Eager R and Nemunaitis J: GM-CSF gene-transduced tumor vaccines. Mol Ther 12: 18-27, 2005.

17. Le DT, Pardoll DM and Jaffee EM: Cellular vaccine approaches. Cancer J 16: 304-310, 2010.

18. Gupta R and Emens LA: GM-CSF-secreting vaccines for solid tumors: moving forward. Discov Med 10: 52-60, 2010.

19. Vonka V: Immunotherapy of chronic myeloid leukemia: present state and future prospects. Immunotherapy 2: 227-241, 2010.

20. Borrello IM, Levitsky HI, Stock W, et al: Granulocytemacrophage colony-stimulating factor (GM-CSF)-secreting cellular immunotherapy in combination with autologous stem cell transplantation (ASCT) as postremission therapy for acute myeloid leukemia (AML). Blood 114: 1736-1745, 2009.

21. Ho VT, Vanneman M, Kim H, et al: Biologic activity of irradiated, autologous, GM-CSF-secreting leukemia cell vaccines early after allogeneic stem cell transplantation. Proc Natl Acad Sci USA 106: 15825-15830, 2009.

22. Zilberberg J, Friedman TM, Dranoff G and Korngold R: Treatment with GM-CSF secreting myeloid leukemia cell vaccine prior to autologous-BMT improves the survival of leukemia-challenged mice. Biol Blood Marrow Transplant 17: 330-340, 2011.
23. Smith BD, Kasamon YL, Kowalski J, et al: K562/GM-CSF immunotherapy reduces tumor burden in chronic myeloid leukemia patients with residual disease on imatinib mesylate. Clin Cancer Res 16: 338-347, 2010.

24. Lucansky V, Sobotkova E, Tachezy R, Duskova M and Vonka V: DNA vaccination against bcr-abl-positive cells in mice. Int $\mathrm{J}$ Oncol 35: 941-951, 2009.

25. Hruskova V, Moravkova A, Babiarova K, Ludvikova V, Fric J, Vonka V and Forstova J: Bcr-Abl fusion sequences do not induce immune responses in mice when administered in mouse polyomavirus based virus-like particles. Int J Oncol 35: 1247-1256, 2009.

26. Petrackova M, Sobotkova E, Duskova M, Jinoch P and Vonka V: Isolation and properties of gene-modified mouse bcr-abl-transformed cells expressing various immunostimulatory factors. Neoplasma 56: 194-201, 2009.

27. McLaughlin J, Chianese $\mathrm{E}$ and Witte $\mathrm{ON}$ : In vitro transformation of immature hematopoietic cells by the P210 BCR/ABL oncogene product of the Philadelphia chromosome. Proc Natl Acad Sci USA 84: 6558-6562, 1987.

28. Sobotkova E, Ludvikova V, Petrackova M, et al: Characteristic of two mouse bcr-abl-transformed cell lines: I. General properties of the cells. Folia Biol (Praha) 51: 12-18, 2005.

29. Jelinek F, Sobotkova E and Vonka V: Characteristics of two mouse bcr-abl-transformed cell lines. II. Pathological lesions induced in mice. Folia Biol (Praha) 51: 93-102, 2005.

30. Smahel M, Sima P, Ludvikova V and Vonka V: Modified HPV16 E7 Genes as DNA Vaccine against E7-Containing Oncogenic Cells. Virology 281: 231-238, 2001.

31. Rossner P, Bubenik J, Sobota V, et al: Granulocyte-macrophage colony-stimulating factor-producing tumour vaccines. Folia Biol (Praha) 45: 173-177, 1999.

32. Zolotukhin S, Potter M, Hauswirth WW, Guy J and Muzyczka N: A 'humanized' green fluorescent protein cDNA adapted for highlevel expression in mammalian cells. J Virol 70: 4646-4654, 1996.

33. Petráčková $\mathrm{M}$, Stanêk L, Mandys V, Dundr P and Vonka V: Properties of bcr-abl-transformed mouse 12B1 cells secreting interleukin-2 and granulocyte-macrophage colony stimulating factor: II. Adverse effects of GM-CSF. Int J Oncol (In press).

34. Chorna I, Bilyy R, Datsyuk L and Stoika R: Comparative study of human breast carcinoma MCF-7 cells differing in their resistance to doxorubicin: effect of ionizing radiation on apoptosis and TGF-beta production. Exp Oncol 26: 111-117, 2004.

35. Davis-Sproul JM, Harris MP, Davidson NE, Kobrin BJ, Jaffee EM and Emens LA: Cost-effective manufacture of an allogeneic GM-CSF-secreting breast tumor vaccine in an academic cGMP facility. Cytotherapy 7: 46-56, 2005.

36. Rosenthal FM, Kulmburg P, Fruh R, et al: Systemic hematological effects of granulocyte colony-stimulating factor produced by irradiated gene-transfected fibroblasts. Hum Gene Ther 7: 2147-2156, 1996.

37. Jaffee EM and Pardoll DM: Considerations for the clinical development of cytokine gene-transduced tumor cell vaccines. Methods 12: 143-153, 1997. 